\title{
Commercial Air Sensor Alarm Systems
}

\author{
Aaron Don M. Africa, Phillip Christian C. Cardino, John Rossi Rafael R. Llaga \\ Department of Electronics and Communications Engineering \\ De La Salle University, Manila \\ 2401 Taft Ave., Malate, Manila 1004, \\ Philippines, aaron.africa@dlsu.edu.ph
}

\begin{abstract}
Polluted air has been a problem for most countries up until now. The pollution caused by modern-day technology has increased throughout the years of development and innovation. Humans have been discovering more advanced technology, but they have yet to find a solution to the common problems. It is well known that air pollution can cause various health issues and that it can be a big threat to the people who are exposed to it for prolonged periods. This study proposes a way to detect air quality inside structures using a commercial air quality sensor alarm system. This system can determine if the air within a certain area is harmful to humans. The group did not consider the total cost of the system if ever it is implemented. The use of it for outdoor purposes was also not considered since the goal of the study is to design an alarm using indoor air quality sensors.
\end{abstract}

Key words: Air pollution, indoor, alarm system, health, sensor, air quality.

\section{INTRODUCTION}

Nowadays, it can be observed that the air quality of air has gone down with time. With the increasing number of automobiles, factories, and other factors that increase air pollution, it can be hypothesized that air quality won't get any better as time passes. The effects of having polluted air an area to one's health can be catastrophic. According to [1], with the current state of China's air quality, it has been inevitable that the residents living there, mostly in the urban areas, acquire diseases that affect the lungs. In their study, they have concluded that most males of low educational or low economic background have higher risks of PM2.5 induced lung cancer caused by air pollution in China.

Lung cancer is not the only disease that can be caused by low air quality. The paper of [2], conducted a study in which they found out the impact of air pollution on incidences of diabetes and survival of those who already have the disease. It is stated in their study that exposure to NO2 particularly is a risk factor for morbidity and mortality related to diabetes mellitus. The results they gathered showed that differences in obesity status suggest that obese young adults are more vulnerable to the harmful effects of air pollution compared to the ones who aren't.
Humans find solutions in order to reduce the amount of pollution they inhale. It may be as simple as wearing a face mask or refusing to buy their own motor vehicle. It is hard to ignore that the air we breathe may not be as good as it was before, but it is still possible to find a solution for it. Inventing modern contraptions to reduce the influence of the polluted air can be a solid foundation on what can be used as a permanent solution for the problem.

\section{BACKGROUND OF THE STUDY}

The research of [3] investigated the presence of nitrogen dioxide as a dynamic influence of lungs and heart disease in the megacity of Iran. The gas is a by-product of smoking cigarettes. However, due to poor regulation and indoor ventilation, it put risk to several people who may be a non-smoker. Sensor alarm systems are important. Some sensors have fuzzy logic systems on them $[4,5]$.

A study made by [6] expands the account of $\mathrm{CO}$ poisoning, having more than 20000 cases of non-lethal poisoning and 400 deaths in the United State in a year. Some of the severe symptoms include paralysis, stroke and permanent brain damage.

The research of [7] discussed the aftermath of less secure household from pollution. It also stated the particle matters (PM) from cooking that deteriorate the health of people in their own home. The research of [8] talks about the effect of concentration of carbon dioxide indoors lead to a bad physical and mental health state.

The paper of [9] mention several particles matters that enter through the lungs in Particle deposition in the human lung: Health implications of particulate matter from different sources. It models the lung similar to a trumpet where the particles are balls of different sizes and density and would affect the performance of the trumpet.

A case where hazardous PM is generated indoors from unmaintained used cooking oil is explained in. Cooking oil is a primary ingredient in several dishes; however, leftover is potentially dangerous in a long time. This applies to other oils [10]. 
The effect of carbon monoxide is further explained and established from the research of [11]. They stated the characteristic of carbon monoxide as an unstable gas, which reacts to several molecules in the body.

The paper of [12] provided the distinct cause-and-effect between abnormalities in the heart and nitrogen dioxide in Data on the short-term effect of nitrogen dioxide on cardiovascular health in Wallonia, Belgium. Admission usually happens in winter, as ventilation and outdoor activities are fewer in the season.

The paper of [13] tackles the long-lasting effect of smog that happens in Kalimantan, Indonesia in 2012 which leads to injuries and death in the region. According to them the region's air quality has declined for years, even affecting people indoors.

\section{STATEMENT OF THE PROBLEM}

Air pollution has been a problem that needs to be taken care of for a long time. It cannot be stressed enough that there are massive health problems that can be caused by inhaling too much-polluted air. Different countries all around the world have different levels of air pollution, factors affecting this are the different ways of living. Most countries that have a lot of factories, usually the 1st world countries have lots of factories which contribute to a lot of air pollution. But this is not what usually causes the air to be polluted, garbage, smoke from cars, and other factors. Most of the time, it is the second and third world countries that contribute to air pollution because of their poor garbage disposal and a large number of automobile owners.

\section{SIGNIFICANCE OF THE STUDY}

This study of Commercial Air Sensor Systems can be a significant help in the maintenance of air quality indoors. This study will also benefit the further implementation of air quality control within different structures. With the help of the proper installment of maintenance in desired structures, an air quality sensor can immediately determine if there is a harmful substance in the air. This is important to the people who work or stay indoors for long periods. They will be notified if the air inside the room is harmful, like toxic gas, etc. Prolonged exposure to air pollution can cause severe health problems. It was mentioned in their articles that obese people are more likely to get ill, usually these subjects get diagnosed with Diabetes. With this, the study will hugely benefit the health industry. It can help with the discovery of a new apparatus to decrease the chances of a person getting ill from air pollution. It cannot be guaranteed to be able to completely erase the threat that it causes, but it can help reduce the possibilities of various other diseases that are caused by it.

\section{DESCRIPTION OF THE SYSTEM}

Since the system is a medium-cost project, it is meant to be moderately efficient as there are several options available already in the market. The framework, however, is considered to be stable as it features a lot of feedback.

An air quality sensor is a device or a component that recognizes the gas on its environment. While there are several kinds of air quality sensor, the types implemented in the system are electrochemical sensors and an infrared sensor.

Electrochemical Sensor is an air quality sensor, with a medium price that uses current to detect specific spectrum of gas. In this system, it is used in detecting oxygen, carbon monoxide and nitrogen dioxide, all of which are invisible gases. It has a good sensing capability, time response and boundaries. A catalyst is the basis of the sensor that the mentioned gases reacts to. The reaction produces current that is sent to the system to be read.

Infrared Sensors is a low-cost sensor that can read carbon dioxide using beams of infrared light. The infrared light is then absorbed and analyzed where it returns the value for the concentration of the gas.

In this system, a sound alarm is used as the response to the sensors. This alerts nearby people of the hazard that is occurring. It has back-up chargeable battery in case of a power outage to supply the sensor for a short time. An LED is attached to notify if the alarm is powered.

In order for the system to initiate, it must be first supplied power. Once the power is supplied, the sensor activates, doing cycles of scanning.

It will only advance when at least one of the three sensors detected anomaly the system considered to be dangerous. Should the current reach a certain point where the system considered it to be hazardous over some time, a switch will be activated to sound the alarm or notify a nearby entity. The hazard limit is breached when the current is higher except for the oxygen sensor, where the current is below expected count over some time.

Only then it will sound the system with the prior condition and does have the power to supply for it. If there is no power present, the LED of the alarm will turn off to notify a need for maintenance to nearby people. Another branch is present to charge the battery. This is in case of an emergency where a power outage happens.

Common electrochemical sensors for oxygen include hydroxyl as a catalyst, while carbon monoxide and nitrogen dioxide have tin oxide or tungsten oxide, as both are reactive to oxygen. Figure 1 shows the flowchart of the system. 


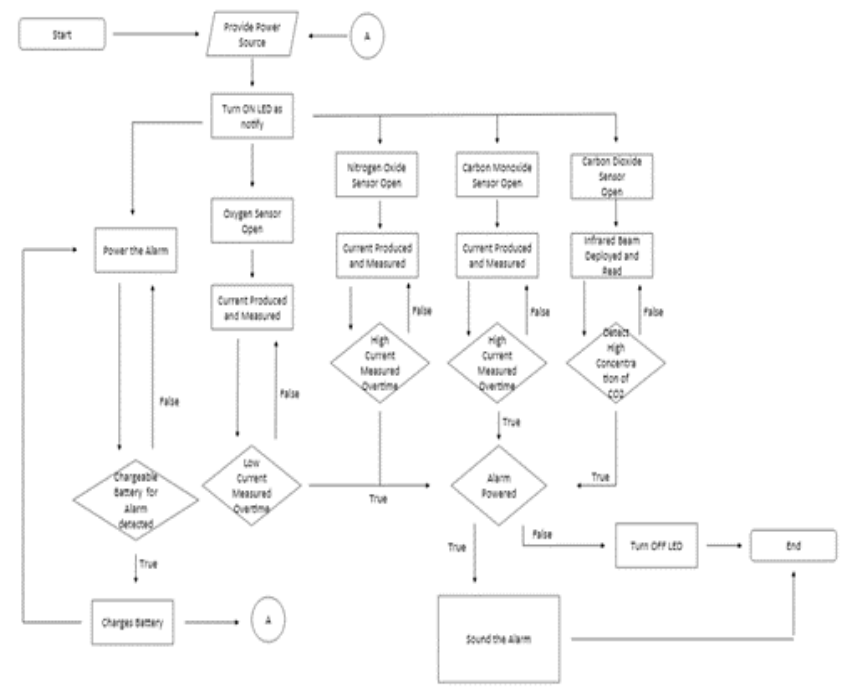

Figure 1: Flow chart of the system

The voltage sources must supply for the sensors as well as the alarm.

\section{REVIEW OF RELATED LITERATURE}

The study of [14] aimed to demonstrate how sensors that are connected as a network can analyze pollutant emission trends at the London Heathrow Airport. Sensor network observations have been used to discern airport emissions unambiguously from long-range transportation, and then to determine emission indices from the different airport operations. The methodology of measurement and analysis is seen in this case study is widely applicable in many sophisticated air quality conditions including the development of megacities, and has a significant potential to educate strategies its initiatives in a cost-effective manner that would reduce possible health effects of air pollution as well as other environmental concerns like greenhouse gas emissions regulations.

Proof of concept apparatus for the design of a simple, low cost, mobile e-nose for real-time victim localization (human presence) based on indoor air quality monitoring sensors is a journal article by [15]. This study proposes that an Electronic nose (E-nose) can be used to collect air samples to prepare, store, and manipulate. The apparatus was aimed to be used for search and rescue operations, and a planned prototype was already prepared to be tested in the future.

This study of [16] proposed a new data fusion called multi-sensor space-time data fusion. Based on the methodology suggested in this report, the authors made micro-sensor data more accurate and enhanced air quality monitoring's higher spatial-temporal resolution. They used the concept of Optimum Linear Data Fusion and implemented this for spatial-temporal approximation with the multi-time phase Kriging procedure.

\section{THEORETICAL CONSIDERATION}

Given that this system is a medium cost, the sensors are likely to be in a moderate spectrum when it comes to efficiency. There are multiple types of cost-efficient sensors that can be added to the system. For example, the existence of micro-air quality sensors capable of identifying a wider spectrum of gases. There are several materials that are in the market. For example, Nafion is used as an oxygen sensor. It is also capable of ozone sensing, which is another dangerous gas if concentrated.

Should the efficiency of materials cover their cost at the minimum with the modus operandi of the system remain unchanged.

\section{DATA AND RESULTS}

The system has few notable problems especially concerning the sensors. The oxygen electrochemical sensor always detects oxygen as it is a common gas, thus the catalyst inside the sensor will be easily drained. It is expected to have a lifespan of no more than 2 years.

Nitrogen dioxide and carbon monoxide are notorious as a lethal agent, which among their characteristics, being invisible is the most defining factor.

The lack of oxygen helps in identifying these kinds of gases, as most of them came due to pressure and force. Carbon dioxide is a visible gas; however, it is a ubiquitous signal for carbon combustion. It also able to notify a large presence of other toxic gases.

\section{CONCLUSION}

The objective of this study is to design a system that can detect if the air in a designated area indoors is harmful or not. The group has decided to use metal-oxide sensors and electrochemical sensors as the basis for their theory. The system's flowchart was constructed as follows: the alarm is powered, the alarms scan the designated area for dangerous substances in the air, if the air is safe, it would start to scan again, but if even one of the sensors detects the harmful amount of these said substances, the whole alarm would turn on to warn nearby people. The substances that are considered to be harmful by these sensors are $\mathrm{NO} 2$ as well as $\mathrm{O} 3$ and PM2.5. Having different sensors within a single alarm helps save space and resources in installing and manufacturing these said alarms. It also helps in programming the system with fewer errors.

Problems in the system would most likely occur since the speed of each sensor is different from each other. Since one sensor would be faster than the other, there would be discrepancies in between each one is activated. This would not likely affect the whole system since the sensors scan for different types of substances that share very little qualities. 
With this, the design of the alarm system is complete with all the necessary functions.

\section{REFERENCES}

[1] H.Guo, Z. Chang, J. Wu and W. Li, "Air pollution and lung cancer incidence in China: Who are faced with a greater effect?'Environment International. Vol. 132, 2019. https://doi.org/10.1016/j.envint.2019.105077

[2] L. Paul, R. Burnett, J. Kwong, P. Hystad, A. Donkelaar, L. Bai, M. Goldberg, E. Lavigne, R. Copes, R. Martin, A. Kopp and H. Chen, "The impact of air pollution on the incidence of diabetes and survival among prevalent diabetes cases,'Environment International. Vol. 134, 2020 . https://doi.org/10.1016/j.envint.2019.105333

[3] H. Saki, G. Goudarzi, S. Jalali, G. Barzegar, M. Farhadi, I. Parseh, S. Geravandi, S. Salmanzadeh, F. Yousefi and M. J. Mohammadi, "Study of relationship between nitrogen dioxide and chronic obstructive pulmonary disease in Bushehr, Iran," Clinical Epidemiology and Global Health. Vol. 8, No. 2, pp. 446-449, 2020. https://doi.org/10.1016/j.cegh.2019.10.006

[4] A.Africa, A.Divino and K.Hartigan-Go, "Fuzzy logic temperature control: A feedback control system implemented by fuzzy logic," International Journal of Emerging Trends in Engineering Research. Vol. 8, No. 5, pp. 1879-1885, 2020. https://doi.org/10.30534/ijeter/2020/66852020

[5] J.R.B. Del Rosario and E. Dadios, "Development of a fuzzy logic-based PV solar tracking system simulated using QT fuzzy engine," Conference on Humanoid, Nanotechnology, Information Technology, Communication and Control, Environment and Management, HNICEM. 2014.

[6] N. Hampson, K. Hauschildt, K. Deru and L. Weaver, "Carbon monoxide poisonings in hotels and motels: The problem silently continues," Preventive Medicine Reports. Vol. 16, 2019. https://doi.org/10.1016/j.pmedr.2019.100975

[7] J. Balmes, "Household Air Pollution from Domestic Combustion of Solid Fuels and Health,"Journal of Allergy and Clinical Immunology. Vol. 143, No. 6, pp. 1979-1987, 2019.

[8] K. Azuma, N. Kagi, U.Yanagi and H. Osawa,"Effects of low-level inhalation exposure to carbon dioxide in indoor environments: A short review on human health and psychomotor performance," Environment International. Vol. 121, pp. 51-56,2018. https://doi.org/10.1016/j.envint.2018.08.059

[9] Q. Deng, L. Deng, Y. Miao, X. Guo and Y. Li, "Particle Deposition in the Human Lung: Health Implications of Particulate Matter from Different Sources," Environmental Research. Vol. 169, pp. 237-245, 2019.

[10] K. Alekseev, A. Alpatov, T. Bondarenko, A. Osipov and A. Stavtsev, "State support for providing agricultural producers with petrol, oil and lubricants," International
Journal of Emerging Trends in Engineering Research. Vol. 7, No. 12, pp. 746-755, 2019. https://doi.org/10.30534/ijeter/2019/037122019

[11]R. Levy, "Carbon monoxide pollution and neurodevelopment: A public health concern," Neurotoxicology and Teratology. Vol. 49, pp. 31-40, 2015.

[12] P. Collart, D. Dubourg, A. Levêque, N. BustosSierra and Y. Coppieters, "Data on short-term effect of nitrogen dioxide on cardiovascular health in Wallonia, Belgium," Data in Brief. Vol. 17, pp.172-179, 2018. https://doi.org/10.1016/j.dib.2017.12.056

[13] Restiatun and E. Suratman, "Effect of injured Acute Respiratory Infection (ARI) and Having Toddler in household to the willingness to pay of Smog Risk Mitigation in District of Pontianak and Pontianak City, West Kalimantan,"Procedia - Social and Behavioral Sciences. Vol. 211,pp. 336-341, 2015.

[14] O. Popoola, D. Carruthers, C. Lad, V. Bright, M. Mead, M.Stettler, J. Saffell andR. Jones, "Use of networks of low-cost air quality sensors to quantify air quality in urban settings," Atmospheric Environment. Vol. 194, pp. 58-70, 2018. https://doi.org/10.1016/j.atmosenv.2018.09.030

[15] A. Anyfantis and S. Blionas, "Proof of concept apparatus for the design of a simple, low cost, mobile e-nose for real-time victim localization (human presence) based on indoor air quality monitoring sensors," Sensing and Bio-Sensing Research. Vol.27,2020.

[16] Y. Chu, P. Xu, Z. Yangand W. Li, "Existing building retrofitting for Indoor PM2.5 Concentration Control on Smog Days: Case study in China," Procedia Engineering. Vol. 205, pp. 3222-3227, 2017. https://doi.org/10.1016/j.proeng.2017.10.290 